\title{
Bone mesenchymal stem cells ameliorate ischemia/reperfusion-induced damage in renal epithelial cells via microRNA-223
}

\author{
Xiaopeng Yuan*, Xiaoping Wang, Chuanbao Chen, Jian Zhou and Ming Han
}

\begin{abstract}
Background: Recent studies have indicated that microRNA-223 (miR-223) plays a role in the tissue-protective effect of mesenchymal stem cells (MSCS). NLR family-pyrin domain containing 3 (NLRP3) was reported to affect a renal ischemia/reperfusion (I/R) injury by exerting a direct effect on the renal tubular epithelium. Therefore, we investigated how miR-223 and NLRP3 might function in kidneys exposed to conditions of ischemia and subsequent reperfusion.

Methods: Hypoxia/reoxygenation (H/R) murine renal tubular epithelial cells (RTECs) were cocultured with either MSCs or hypoxia-pretreated MSCs (htMSCs), after which the RTECs were examined for their viability and evidence of apoptosis. Next, miR-223 expression in the MSCs was downregulated to verify that MSCs protected RTECS via the transport of miR-223. Kidney I/R KM/NIH mouse models were created and used for in vivo studies.
\end{abstract}

Results: The results showed that coculture with MSCs significantly increased the viability of RTECs and decreased their rates of apoptosis. The levels of hepatocyte growth factor (HGF), insulin-like growth factor-1 (IGF-1), transforming growth factor beta (TGF- $\beta$ ), and vascular endothelial growth factor (VEGF) in samples of coculture supernatants were higher than those in samples of non-coculture supernatants. A bioinformatics analysis revealed a targeting relationship between miR-223 and NLRP3. A dual luciferase assay showed that miR-223 inhibited NLRP3 expression. The htMSCs displayed a protective function associated with an upregulation of miR-223 as induced by Notch1 and the downregulation of NLRP3. Conversely, inhibition of miR-223 impeded the protective effect of MSCs. In the I/R mouse models, injection of either MSCs or htMSCs ameliorated the damage to kidney tissue, while suppression of miR-223 expression in MSCs reduced their protective effect on mouse kidneys.

Conclusions: Our results demonstrate that miR-223 and NLRP3 play important roles in the treatment of renal tissue injuries with transplanted MSCs.

Keywords: Ischemia/reperfusion, Mesenchymal stem cell, microRNA-223, NLR family-pyrin domain containing 3, Inflammation

\footnotetext{
*Correspondence: transplantyuan@163.com

Third Division of Organ Transplant Center, Eastern Campus of First Affiliated

Hospital, Sun Yat-sen University, Guangzhou 510700, People's Republic of

China
} 


\section{Background}

Renal blood flow is always temporarily disrupted during various surgical treatments, including those performed for the purposes of kidney transplantation, partial nephrectomy, aortic bypass, and treatment of sepsis [1-4]. Although the re-initiation of blood flow to ischemic kidney regions restores their function, the restoration process itself can further damage kidney tissue, resulting in an ischemia/reperfusion (I/R) injury [5]. Patients who are hospitalized due to acute renal failure caused by an $\mathrm{I} / \mathrm{R}$ injury have high rates of morbidity and mortality. In recent years, considerable effort has being made to improve the treatment of renal I/R injuries, and a variety of agents and growth factors have been proven effective for treating these disorders. However, most of these treatment modalities were validated in animal models, and no significant efficacy was demonstrated in human clinical trials [6]. Therefore, the development of novel and effective strategies for managing and treating renal $I / R$ injuries remains a high priority.

In recent years, several studies have demonstrated that mesenchymal stem cells (MSCs) may help attenuate or prevent an $I / R$ injury in multiple organs, including the kidney, and may exert that effect via paracrine/autocrine mechanisms or transdifferentiation into local cell types [7-10]. MSCs are multipotent cells capable of differentiating into various types of specialized mesenchymal cells such as osteoblasts, chondrocytes, adipocytes, and tenocytes [11]. MSCs are easier to culture than embryonic stem cells (ESCs) and other adult stem cells [8], and their functional properties make them promising candidates for use in tissue engineering studies and as agents for treating various diseases, including renal I/ $\mathrm{R}$ injuries [11]. Semedo et al. [12] showed that MSCs could modulate $I / R$ injuries in renal tissue by inhibiting inflammatory responses. Rosova et al. [13] proposed that the beneficial effects of MSCs could be primarily attributed to their ability to mediate complex paracrine actions. Moreover, MSCs were shown to improve the recovery of $I / R$-injured rodents even if they were infused starting at $24 \mathrm{~h}$ after the injury, which suggests that MSCs actively participate in repairing I/R-injured tissues [13-15].

The mechanism by which MSCs protect kidney tissue is complicated, and may involve the paracrine/autocrine effects of cytokines, their transdifferentiation into the local cell type, and/or immunomodulation [8, 16]. A previous study showed that administration of MSCs into a DCD rat kidney alleviated kidney tissue damage [17]. Moreover, recent evidence suggests that the NLR familypyrin domain containing 3 (NLRP3) inflammasome may upregulate cytokine production and play a critical role in renal injuries [18]. NLRP3 may also contribute to renal $\mathrm{I} / \mathrm{R}$ injuries by directly affecting the renal tubular epithelium [19]. Given that NLRP3 is negatively regulated by microRNA-223 (miR-223) [20], an enhanced expression of miR-223 might be expected to ameliorate the damage to renal tissues. The cardioprotective effect of MSCs is mediated through exosomal miR-223. Moreover, Notch receptors expressed in MSCs were proven to be upstream regulators of miR-223 [21, 22]. Therefore, we hypothesized that MSCs might exert their protective function by suppressing NLRP3 via Notchinduced activation of miR-223. We then tested our hypothesis in a series of in vitro and in vivo assays.

The effect of MSC administration and the role of miR223/NLRP3 signaling in renal I/R injuries were examined using hypoxia/reoxygenation (H/R) murine renal tubular epithelial cells (RTECs), and were then verified in a renal $\mathrm{I} / \mathrm{R} \mathrm{KM} / \mathrm{NIH}$ mouse model. Our results not only verified the protective effect of MSCs on kidney tissue, but helped explain the mechanism by which MSCs limit the extent of $\mathrm{I} / \mathrm{R}$ injuries.

\section{Methods \\ Preparation of cells}

Murine RTECs were purchased from Zhongqiaoxinzhou Cell Research (Cat. No. M4100; Shanghai, China) and cultured in Dulbecco's modified Eagle's medium (DMEM) supplemented with $10 \%$ fetal bovine serum (FBS) and penicillin/streptomycin at $37{ }^{\circ} \mathrm{C}$ in a $5 \% \mathrm{CO}_{2}$ atmosphere. MSCs were generated from bone marrow tissue extracted from the femurs of sacrificed mice, and suspended in sterile phosphate-buffered saline (PBS). The extracted cells were then resuspended in DMEM, filtered through $70-\mu \mathrm{m}$ mesh lattice, and plated in flasks containing DMEM supplemented with 10\% FBS (Thermo Fisher Scientific, Waltham, MA, USA). After $72 \mathrm{~h}$ of culture, any nonadherent cells were removed, and the adherent cells were passed at a low density into new flasks. Cells that displayed a typical spindle-shaped appearance were used for subsequent assays.

\section{Induction of the H/R RTEC model and coculture}

To detect the effect of MSCs on miR-223/NLRP3 activity, the RTECs were divided into the following four groups: 1) RTEC group (normal RTECs); 2) HR RTEC group, (H/R-treated RTECs); 3) HR RTEC + MSC group (H/R-treated RTECs cocultured with MSCs for $48 \mathrm{~h}$ ); and 4) HR RTEC + htMSC group (H/R-treated RTECs cocultured with hypoxia-pretreated MSCs (htMSCs) for $48 \mathrm{~h}$ ).

To detect the role of the miR-223/NLRP3 pathway in renal I/R injuries, the RTECs were divided into the following two groups: 1) Negative control group (H/Rtreated RTECs cocultured with MSCs transfected with negative control inhibitors); and 2) miR-223 inhibitor 
group (H/R-treated RTECs cocultured with MSCs transfected with a miR-223-specific inhibitor).

For the H/R treatment, RTECs were plated into six-well plates and exposed to $\mathrm{H} / \mathrm{R}$ conditions as previously described [23]. Briefly, the RTECs were inoculated at concentrations of $5 \times 10^{5}$ cells per well and incubated in DMEM culture medium containing 10\% FBS. To synchronize cell growth, the culture medium waschanged to serum-free DMEM at $24 \mathrm{~h}$ before treatment. Next, the ischemia/hypoxia medium (glucose; $10,000 \mathrm{mg} / \mathrm{L}$ ) was added to the cells, and the plates were put into doublelayered, sealed, self-styled bags with an anaerobic indicator. The bags were then filled with low-oxygen gas $(5 \%$ $\mathrm{CO}_{2}, 95 \% \mathrm{~N}_{2}$ ) to expel the air. When the purple anaerobic indicator turned red, the bag was sealed to maintain the anaerobic condition for $2 \mathrm{~h}$, after which oxygen was added to the bag and the medium was changed to DMEM with FBS for further incubation. Hypoxia treatment has been reported to improve the therapeutic efficacy of MSCs [13]. For hypoxia pretreatment of MSCs, the cells were cultured in 12-well plates with DMEM supplemented with $10 \% \mathrm{FBS}, 50 \mu \mathrm{g} / \mathrm{mL}$ penicillin, and $50 \mathrm{mg} / \mathrm{L}$ gentamicin (Sigma, St. Louis, MO, USA) prior to being cocultured with RTECs. Each parameter examined in the following assays was measured in at least three identical samples. A double-chamber coculture system was used for cell coculture. The system was separated into upper and lower chambers by a $0.4-\mu \mathrm{m}$ pore-size membrane (12-well insert; BD Biosciences. Franklin Lakes, NJ, USA). RTECs $\left(1 \times 10^{5}\right.$ cells/well $)$ were cultured in the lower chamber and MSCs or htMSCs $\left(1 \times 10^{5}\right.$ cells/well $)$ were cultured in the upper chamber. Cells were harvested after $24 \mathrm{~h}, 48 \mathrm{~h}$, and $72 \mathrm{~h}$ of culture, respectively.

\section{Creation of renal I/R mouse models and transplantation of MSCs}

Twenty-four $\mathrm{KM} / \mathrm{NIH}$ mice (female, $34 \pm 2.1 \mathrm{~g}$ ) were used for in vivo assays and were randomly assigned to four different groups (six mice per group): 1) Control group (mice underwent I/R treatment and were abdominally intravenously injected with the same volume of normal saline as used for negative control groups); 2) MSC group (15 min before I/R treatment the mice were abdominally intravenously injected with $2 \times 10^{6}$ MSCs in a volume of $200 \mu \mathrm{L}$ and then transfected with a negative control inhibitor); 3) miR-223 inhibitor group (15 min before $I / R$ treatment the mice were abdominally intravenously injected with $2 \times 10^{6}$ miR-223 knockdown MSCs); and 4) htMSC group (15 min before I/R treatment the mice were abdominally intravenously injected with $2 \times 10^{6}$ hypoxia-pretreated MSCs). For induction of the renal I/R model, mice were anesthetized with isoflurane and their rectal temperature was maintained at $37{ }^{\circ} \mathrm{C}$. After a midabdominal laparotomy, the kidneys were exposed and the renal pedicles were clamped with atraumatic vascular clamps for $60 \mathrm{~min}$. While the clamps were applied, the left carotid artery was cannulated with PE-50 tubing to allow for intraaortic cell delivery immediately after blood flow was reestablished. All mice were sacrificed at $24 \mathrm{~h}$ after their operation.

\section{Transfections of MSCs}

MSCs $\left(1 \times 10^{5}\right)$ were seeded into six-well plates (18 replications). Transfection or hypoxic stimulation was performed until the cells reached $70-80 \%$ confluence. A miR-223-specific inhibitor (sequence: 5 '-ACAGUCAAA CAGUUUAUGGGUU-3') or negative control (sequence: 5'-UGCGCUGCUGGUGCCAACCCUAUUCU-3') purchased from Jikai Biosciences (Shanghai, China) was transfected into the MSCs using Lipofectamine 2000 reagent (Thermo Fisher Scientific Waltham, MA, USA) according to the manufacturer's instructions. At $24 \mathrm{~h}$ posttransfection, the MSCs were harvested and used for coculture with RTECs. The knockdown effect in the MSCs lasted for $96 \mathrm{~h}$.

\section{Dual luciferase assay}

Full length NLRP3 mRNA (mNLRP3) 3'-untranslated region (UTR) was inserted into the psiCHECK-2 luciferase reporter vector to construct psiCHECK-2-mNLRP3wt. The mutated form of mNLRP3 3'-UTR was used to construct psiCHECK-2-mNLRP3-mut. ViaFect ${ }^{\text {mox }}$ Transfection Reagent (Promega, Fitchburg, WI, USA) was used to cotransfect $1 \mu \mathrm{g}$ of psiCHECK-2-mNLRP3-wt or psiCHECK-2-mNLRP3-mut with $50 \mathrm{~nm} / \mathrm{L}$ miR-223 mimic or miR-223 inhibitor into RTECs. Dual luciferase activity was assayed $48 \mathrm{~h}$ later.

\section{CCK-8 assay}

The cell counting kit-8 (CCK-8) assay was used to detect RTEC proliferation in the different groups. Briefly, RTECs in the coculture system were harvested at $24 \mathrm{~h}$, $48 \mathrm{~h}$, and $72 \mathrm{~h}$, respectively, after which $100 \mu \mathrm{L}$ of CCK8 solution (Dojindo Molecular Technologies, Gaithersburg, MD, USA) was added to each well. After $4 \mathrm{~h}$ of incubation at $37{ }^{\circ} \mathrm{C}$ the optical density of each well at $450 \mathrm{~nm}$ was read with a microplate reader (Thermo Plate, Rayto Life and Analytical Science C. Ltd., Shenzhen, Guangdong, China).

\section{Flow cytometry assay}

The apoptotic rates of RTECs sampled at $48 \mathrm{~h}$ in the different groups were determined by flow cytometry. Cells in different groups were collected after centrifugation at $600 \mathrm{~g}$ for $5 \mathrm{~min}$, and their apoptotic rates were measured using an Annexin V-FITC Apoptosis Detection Kit (WLA001c, Wanleibio, Shenyang, China) according to 
instructions provided by the manufacturer. Briefly, $5 \mu \mathrm{L}$ of Annexin $\mathrm{V}$ was added to different wells containing RTECs. After a 10-min incubation with Annexin V at room temperature, the cells were resuspended in $1 \times$ binding buffer, and $5 \mu \mathrm{L}$ of propidium iodide was added to each sample. Next, the apoptotic rates were analyzed by flow cytometry (Accuri C6, BD, San Jose, CA, USA). The overall apoptotic rate (UR + LR-all apoptosis cell percentage) was equal to the sum of the late apoptotic rate (UR, upper right quadrant-advanced stage apoptosis cell percentage) and the early apoptotic rate (LR, lower right quadrant-prophase apoptosis cell percentage).

\section{Enzyme-linked immunosorbent assay}

The levels of renal protecting cytokines, including hepatocyte growth factor (HGF; Cat. No. E-EL-R0496c, Elabscience-Biotech Co. Ltd., Wuhan, China), transforming growth factor beta (TGF- $\beta$; Cat. No. CSB-E04727r, Cusabio Biotech, Wuhan, China), insulin-like growth factor-1 (IGF-1; Cat. No. CSB-E04582r, Cusabio Biotech, Wuhan, China), and vascular endothelial growth factor (VEGF; Cat. No. CSB-E04757r, Cusabio Biotech, Wuhan, China) were measured using enzyme-linked immunosorbent assay (ELISA) kits according to the manufacturers' instructions. Serum blood urea nitrogen (BUN) levels were detected using an ELISA kit manufactured by Bio-Medical Assay, Beijing, China.

\section{Quantitative real-time polymerase chain reaction (qRT-PCR)}

The total RNA in different cell samples at $48 \mathrm{~h}$ was extracted using an RNA Purified Total RNA Extraction Kit according to the manufacturer's instructions (Cat. No. RP1201, BioTeke, Beijing, China). $\beta$-actin and $U 6$ were used as internal reference genes. Next, Super M-MLV reverse transcriptase (Cat. No. RP6502, BioTeke, Beijing, China) was used to reverse transcribe samples of total RNA into cDNA templates. Each $20 \mu \mathrm{L}$ RT-PCR reaction mixture consisted of $10 \mu \mathrm{L}$ Bestar ${ }^{\circ}$ SybrGreen qPCR Master Mix, $0.5 \mu \mathrm{L}$ of each primer (miR-223, forward: 5'-ACACTCCAGCT GGGTGTCAGTTTGTCAAATAC-3', universally reverse: 5' -CTCAACTGGTGTCGTGGA-3'; NLRP3, forward: 5' CAGAAGGCTGTGAGGGGAGA-3', reverse: 5'-GCAG ACCAGGGGGATGAAG-3'; Notch1, forward: 5' -GAGA TTGGCTCCTATCGCTG-3', reverse: $5^{\prime}$-GGGCAGTCA TCCACATTTTC-3'; Bcl-2, forward: 5'-GGCATCTTCT CCTTCCAGC-3', reverse: 5'-CCTCCCCCAGTTCACC C-3'; Bcl-XL, forward: 5'-TCGCCAGCCTCTCTCA GC-3', reverse: 5'-AGACCCCCAGTGCCATCA-3'; Caspase-1, forward: 5'-CCTCAAGTTTTGCCCTTTA GA-3', reverse: 5'-TACGAGTGGGTGTTTTCATTATT3'; Caspase-3, forward: 5'-GGGTGCGGTAGAGTAAGC A-3', reverse: 5'-GGAACGAACGGACCTGTG-3'; $\beta$ - actin forward: 5'-GGAGATTACTGCCCTGGCTCCTA3', reverse: 5' -GACTCATCGTACTCCTGCTTGCTG-3'; U6 forward: 5'-CTCGCTTCGGCAGCACA-3', reverse: 5'-AACGCTTCACGAATTTGCGT-3'), $2 \mu \mathrm{L}$ cDNA template, and $7 \mu \mathrm{L}$ Rnase-free $\mathrm{H}_{2} \mathrm{O}$. The amplification parameters were as follows: denaturation at $95{ }^{\circ} \mathrm{C}$ for $2 \mathrm{~min}$, followed by 40 cycles at $94{ }^{\circ} \mathrm{C}$ for $20 \mathrm{~s}, 58^{\circ} \mathrm{C}$ for $20 \mathrm{~s}$, and $72{ }^{\circ} \mathrm{C}$ for $30 \mathrm{~s}$, after which the reaction was stopped by lowering the temperature to $4{ }^{\circ} \mathrm{C}$ for $5 \mathrm{~min}$. Relative expression levels of the targeted molecules were calculated by an Exicycler ${ }^{\text {mi }} 96$ PCR system (Bioneer, Alameda, CA, USA) using the $2^{-\triangle \Delta \mathrm{ct}}$ method.

\section{Western blot assays}

The total proteins in different groups were extracted using a total protein extraction kit according to the manufacturer's instructions (Cal. No. WLA019, Wanleibio, China). GAPDH was used as an internal reference. The protein concentration in each sample was determined using the BCA method. A $20-\mu \mathrm{L}$ aliquot of protein $(40 \mu \mathrm{g})$ was separated by electrophoresis on a $10 \%$ sodium dodecylsulfate polyacrylamide gel. Following separation, the targeted proteins were transferred onto polyvinylidene difluoride (PVDF; BD, San Jose, CA, USA) sheets, which were then washed with TTBS for $5 \mathrm{~min}$ prior to being incubated in a powdered skim milk solution for $1 \mathrm{~h}$. Primary antibodies against NLRP3 (1:400, Santa Cruz Biotechnology, Santa Cruz, CA, USA), Notch1 (1:1500, Santa Cruz Biotechnology Santa Cruz, CA, USA), Bcl-2 (1:500, Santa Cruz Biotechnology, Santa Cruz, CA, USA), Bcl-XL (1:1000, Santa Cruz Biotechnology, Santa Cruz, CA, USA), caspase-1 (1:2000, Abcam, Cambridge, MA, USA), caspase-3 (1:800, Abcam, Cambridge, MA, USA), and glyceraldehyde-3-phosphate dehydrogenase (GAPDH; 1:4000, Abcam; Cambridge, MA, USA) were incubated with the membranes at $4{ }^{\circ} \mathrm{C}$ overnight. Following incubation, the membranes were washed four times with TTBS, after which secondary HRP goat anti-rabbit antibodies (1:20,000, Cat. No. BA1054, Wuhan, China) were added to the mixture and incubated with the membranes for $45 \mathrm{~min}$ at $37{ }^{\circ} \mathrm{C}$. After an additional six washes with TTBS, the blots were developed using Beyo ECL Plus reagent, and the results were detected with a gel imaging system. The relative levels of BDNF expression in the different samples were calculated using Gel-Pro-Analyzer (Media Cybernetics, Rockville, MD, USA).

\section{Immunofluorescence assays}

RTECs which had received the different respective treatments for $48 \mathrm{~h}$ were seeded into 14-well chambers, washed with PBS, and fixed with $4 \%$ paraformaldehyde for $15 \mathrm{~min}$. The cells were then permeabilized with $0.5 \%$ Triton X-100 for $30 \mathrm{~min}$. Next, the cells were washed 
three times with PBS (5 min per wash), and blocked with $10 \%$ goat serum for $15 \mathrm{~min}$. Primary rabbit polyclonal antibodies to Notch1 (1:200, Santa Cruz Biotechnology, USA) and NLRP3 (1:100, Santa Cruz Biotechnology, USA) were then added, and the cells were incubated overnight at $4{ }^{\circ} \mathrm{C}$ in $1 \%$ goat serum. The cells were then incubated and stained with a fluorescein isothiocyanate secondary antibody (goat-anti-rabbit-Alexa 594 conjugated antibodies, Life Technologies, USA) for $1 \mathrm{~h}$. Following incubation with the secondary antibody, the cells were washed and then stained with 4,6-diamino-2-phenyl indole (DAPI; Life Technologies, USA) for $5 \mathrm{~min}$ at room temperature. After three 5min washes with PBS buffer, the slides were fixed and imaged with a fluorescence microscope at $\times 100$ magnification.

\section{Hematoxylin and eosin (H\&E) staining}

The histological changes in sections of kidney tissue from the different groups were observed using $H \& E$ staining. Briefly, the tissues were placed into Bouin's solution ( $4 \%$ formaldehyde) for perfusion fixation, after which they were dehydrated using different concentrations of alcohol and vitrified in dimethylbenzene. The tissues samples were then embedded in paraffin, sectioned, stained with $H \& E$, and examined under a microscope at $\times 200$ magnification. Following H\&E staining, the cell nuclei were stained blue and cytoplasmic areas were stained red.

\section{Periodic acid-silver methenamine (PASM) staining}

PASM staining was used as an optimal method for identifying the extracellular matrix in sections of renal tissue. Briefly, tissues sections were stained in $1 \%$ acid periodic, after which they were treated with an Ag-methenamine solution and stained with $\mathrm{AuCl}$. After staining, collagen fibers appeared as dark strands against a pink background.

\section{Terminal deoxynucleoitidyl transferase-mediated nick-end labeling (TUNEL) staining}

The rates of cellular apoptosis in samples of renal tissue were determined using TUNEL staining. Briefly, tissue sections were permeabilized with $50 \mu \mathrm{L} 0.1 \%$ Triton X-100 at room temperature for $8 \mathrm{~min}$. Next, the sections were washed three times with PBS buffer ( 5 min per wash) and then incubated in $3 \% \mathrm{H}_{2} \mathrm{O}_{2}$ for $10 \mathrm{~min}$ at room temperature. After another three 5min washes in PBS buffer, the sections were covered with TUNEL reaction solution and incubated at $37{ }^{\circ} \mathrm{C}$ for $1 \mathrm{~h}$ in a darkened humidified chamber. Following incubation, the sections were washed three times with PBS buffer (5 min per wash), and then incubated with Converter-POD at $37{ }^{\circ} \mathrm{C}$ for $30 \mathrm{~min}$. The reaction was then stopped, and the cells were restained with hematoxylin. The stained renal tissues were then examined under a microscope (CX41, Olympus, Shinjuku, Tokyo, Japan) at $\times 200$ magnification.

\section{Immunohistochemical detection methods}

Sections prepared from different tissue samples were examined independently by senior pathologists, and then immediately frozen for Notch1 detection. Tissue slides to be used for immunohistochemical assays were stored at $60{ }^{\circ} \mathrm{C}$ overnight, and then incubated with dimethylbenzene for dewaxing. The slides were then hydrated with different concentrations of alcohol (100\% for $5 \mathrm{~min}, 95 \%$ for $5 \mathrm{~min}, 85 \%$ for $5 \mathrm{~min}$, and $75 \%$ for $5 \mathrm{~min}$ ) and washed with $\mathrm{H}_{2} \mathrm{O}_{2}$ for $5 \mathrm{~min}$. Slides with tissue sections from different animals were fixed using a methanol solution containing $3 \% \mathrm{H}_{2} \mathrm{O}_{2}$, and then blocked with $1 \%$ bovine serum albumin (BSA) for $30 \mathrm{~min}$ at $37{ }^{\circ} \mathrm{C}$. They were then incubated at $37{ }^{\circ} \mathrm{C}$ for $30 \mathrm{~min}$ with a primary antibody against Notch1 (1:200, Abcam, Cambridge, MA, USA), before being incubated at $4{ }^{\circ} \mathrm{C}$ overnight. After four cycles of washing in $0.01 \mathrm{M}$ PBS (5 min per cycle), a secondary antibody (goat-anti-rabbit-Alexa 594 conjugated antibody; Thermo Fisher Scientific, Waltham, MA, USA) was added to the slides, which were then incubated at $37{ }^{\circ} \mathrm{C}$ for $30 \mathrm{~min}$. After another four cycles of washing in PBS, DAB was added to the slides and reacted for 3-10 $\mathrm{min}$ until the reaction was stopped by addition of $\mathrm{ddH}_{2} \mathrm{O}$. The slides were then restained with hematoxylin, dehydrated, and examined under a microscope at $\times 200$ magnification.

\section{Creatinine clearance rate test}

Urine was collected within $24 \mathrm{~h}$ after each operation and the creatinine levels in plasma and urine samples were detected with a creatinine assay kit (BioAssay Systems, Hayward, CA, USA). The creatinine clearance rate (Ccr) was calculated according to the Cockcroft-Gault formula: (urinary volume $(\mathrm{mL}, 24 \mathrm{~h}) \times$ urinary creatinine $(\mu \mathrm{mol} / \mathrm{L})) /($ serum creatinine $(\mu \mathrm{mol} / \mathrm{L}) \times$ urine collection length $(\min ))$.

\section{Statistical analysis}

All the data are expressed as means \pm SD. One-way analysis of variance (ANOVA), ANOVA for repeated measurements, and Duncan's post-hoc test were all performed using a general linear model. A $P$ value $<0.05$ indicated statistical significance. All the statistical analyses were performed using SPSS Statistics for Windows, version 19.0 (IBM, Armonk, NY, USA). 


\section{Results}

Coculture of RTECs with MSCs increased the viability of RTECs and suppressed their rates of apoptosis

$H / R$ treatment of RTECs decreased their viability and enhanced their rates of apoptosis (Fig. 1), while coculture with MSCs attenuated the effects of $H / R$. When examined at $72 \mathrm{~h}$, the difference in optical density $\left(\mathrm{OD}_{450}\right)$ values between H/R RTECs that were cocultured with MSCs and H/R RTECs that were not cocultured with MSCs was statistically significant $(P<0.05$; Fig. 1a). Similar differences were detected in the apoptosis rates of the two groups of cells (Fig. 1b). Moreover, the cellular activity of RTECs treated with htMSCs at $72 \mathrm{~h}$ was higher than that of RTECs treated with normal MSCs. This suggested that coculture with MSCs can protect RTECs from H/R-induced injuries, and hypoxia pretreatment of MSCs enhanced their protective effect.

\section{Expression of miR-223 was upregulated in RTECs cocultured with MSCs and htMSCs}

The injuries induced by $H / R$ decreased the miR-223 levels in RTECs (Fig. 2a). However, hypoxia treatment increased the levels of miR-223 expression in both MSCs (Fig. 2b) and RTECs (Fig. 2a). The levels of excreted protective cytokines HGF, IGF-1, TGF- $\beta$, and VEGF in the supernatants of coculture medium were significantly increased by treatment with MSCs when compared with those in the RTECs and HR RTECs groups $(P<0.05$; Fig. 3a). Furthermore, the decreased expression levels of all the antiapoptosis indicators (including Bcl-2 and Bcl$\mathrm{XL}$ ) in the HR RTEC groups were restored after treatment with htMSCs (Fig. 3b and c), while the expression levels of proapoptosis indicators (including caspase-1 and caspase-3) were partially downregulated. The results obtained after administration of MSCs indicated that the MSCs had actively contributed to increasing the levels of miR-223 in H/R RTECs.

\section{Notch signaling in MSCs was initiated by hypoxia pretreatment}

The prosurvival mechanism of MSCs was further investigated at the molecular level. A bioinformatics analysis verified existence of a complementary binding site between miR-223 and the 3'-UTR of NLRP3 mRNA (Fig. 4a). A dual luciferase assay showed that treatment with the miR-223 mimic significantly decreased the relative amount of luciferase activity (Fig. 4b), indicating that miR-223 exerted a regulatory effect on NLRP3 mRNA. We also assessed the levels of NLRP3 expression in RTECs obtained from the different groups. NLRP3 expression in RTECs was first induced by H/R treatment and then suppressed in the cocultured groups (Fig. 4c and $\mathrm{d}$ ), and this pattern was synchronized with corresponding changes in cell viability and apoptosis. The

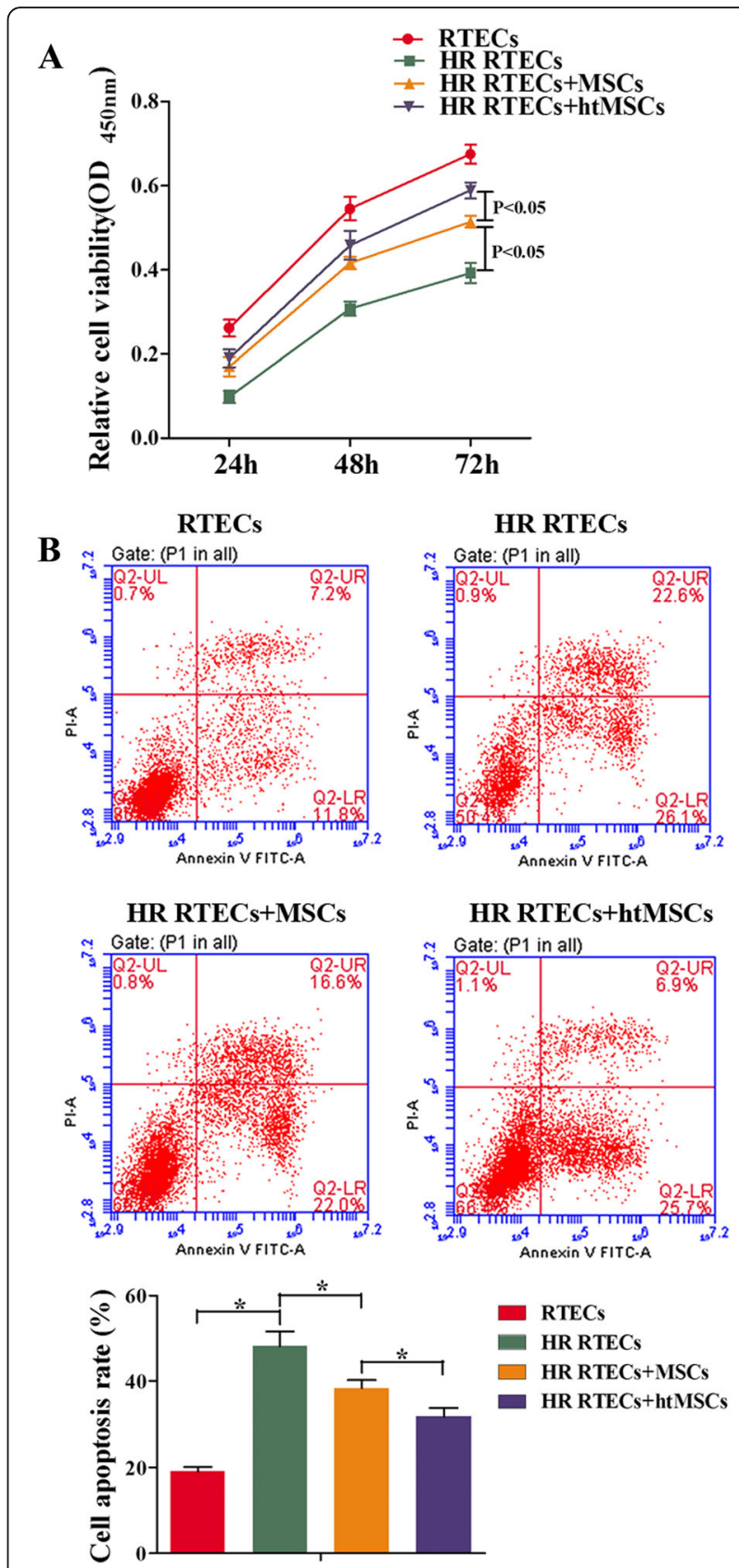

Fig. 1 Coculture with mesenchymal stem cells (MSCS) increased cell viability and suppressed apoptosis of renal tubular epithelial cells (RTECS). RTECs were treated with hypoxia/reoxygenation (HR) stimulation and then cocultured with MSCs or hypoxia-pretreated MSCs (htMSCs) in a double-chamber. RTECs were cultured in the lower chamber and MSCs or htMSCs were cultured in the upper chamber. a RTEC viability was measured with the CCK-8 assay. b Representative images of apoptotic RTECs at $48 \mathrm{~h}$ as detected by flow cytometry. ${ }^{*} P<0.05, n=3$. OD optical density, $P$ I propidium iodide

promoting role of NLRP3 in an acute renal injury was negatively regulated by miR-223. Because miR-223 secreted by MSCs is known to be transferred to RTECs via a paracrine mechanism, we investigated the levels of 


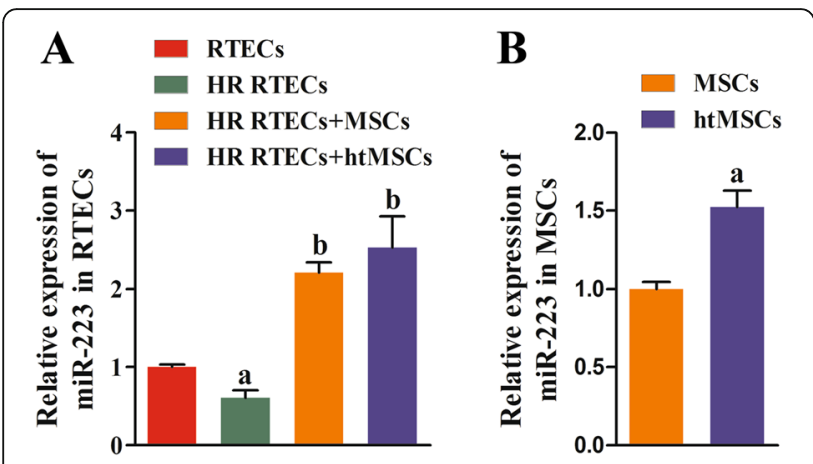

Fig. 2 Expression of miR-223 was upregulated in renal tubular epithelial cells (RTECS) cocultured with mesenchymal stem cells (MSCs) or hypoxia-pretreated MSCs (htMSCs). RTECs were treated with hypoxia/reoxygenation (HR) stimulation and then cocultured with MSCs or htMSCs in a double-chamber. Cells were harvested at $48 \mathrm{~h}$. a Expression of miR-223 in RTECs was detected by qRTPCR. ${ }^{a} P<0.05$, versus the RTEC group; ${ }^{b} P<0.05$, versus the HR RTEC group. $\mathbf{b}$ Expression of miR-223 in MSCs was detected by qRT-PCR. ${ }^{a} P<0.05$, versus the normal cultured MSCs

Notch1 expression in MSCs and found that Notch1 expression was significantly increased by hypoxia pretreatment $(P<0.05$; Fig. $4 \mathrm{e}$ and $\mathrm{f})$. This suggests that miR-223 in MSCs might be upregulated by Notch signaling.

miR-223 contributed to the attenuation of $H / R$ injuries in RTECs by suppressing NLRP3 expression

To verify that miR-223/NLRP3 signaling plays a key role in the kidney protective function of MSCs, we specifically inhibited miR-223 expression in MSCs. We found that the viability of cocultured RTECs became dramatically decreased concomitant with miR223 inhibition $(P<$ 0.05; Fig. 5a), and the rates of RTEC apoptosis were accelerated (Fig. 5b). At the same time, the levels of cell protective cytokines (i.e., HGF, IGF-1, TGF- $\beta$, and VEGF) in the coculture supernatants declined (Fig. 6a). Moreover, the levels of antiapoptosis indicators also declined due to the knockdown of miR-223, while the expression levels of molecules known to promote apoptosis (i.e., caspase-1 and caspase-3) increased (Fig. 6b and c). The downregulation of miR-223 in MSCs induced NLRP3 expression, indicating that miR-223 plays a key role in the mechanism by which MSCs protect kidney tissue (Fig. 7a and b).

\section{In vivo implantation of MSCs ameliorated kidney $\mathrm{I} / \mathrm{R}$ injuries via miR-223-mediated NLRP3 suppression}

To verify our findings in the H/R RTEC model, an in vivo renal $I / R$ injury model was created using $\mathrm{KM} / \mathrm{NIH}$ mice. Samples of kidney tissue from the treated mice were collected for RNA extraction. Next, qRT-PCR was performed to measure the mRNA levels of several cytokines. Mice injected with MSCs or htMSCs had increased levels of cell protective cytokines (HGF, IGF-1, TGF- $\beta$, and VEGF). The levels of those cytokines in mice injected with miR-223 knockdown MSCs were significantly lower than those in both the I/R + MSC and I/ $\mathrm{R}+$ htMSC groups $(P<0.05)$. However, even though the miR-223 levels in MSCs had been reduced, the miR-233 levels in the $I / R+$ MSC group were still higher than those in the I/R group (Fig. 8a). These results confirmed

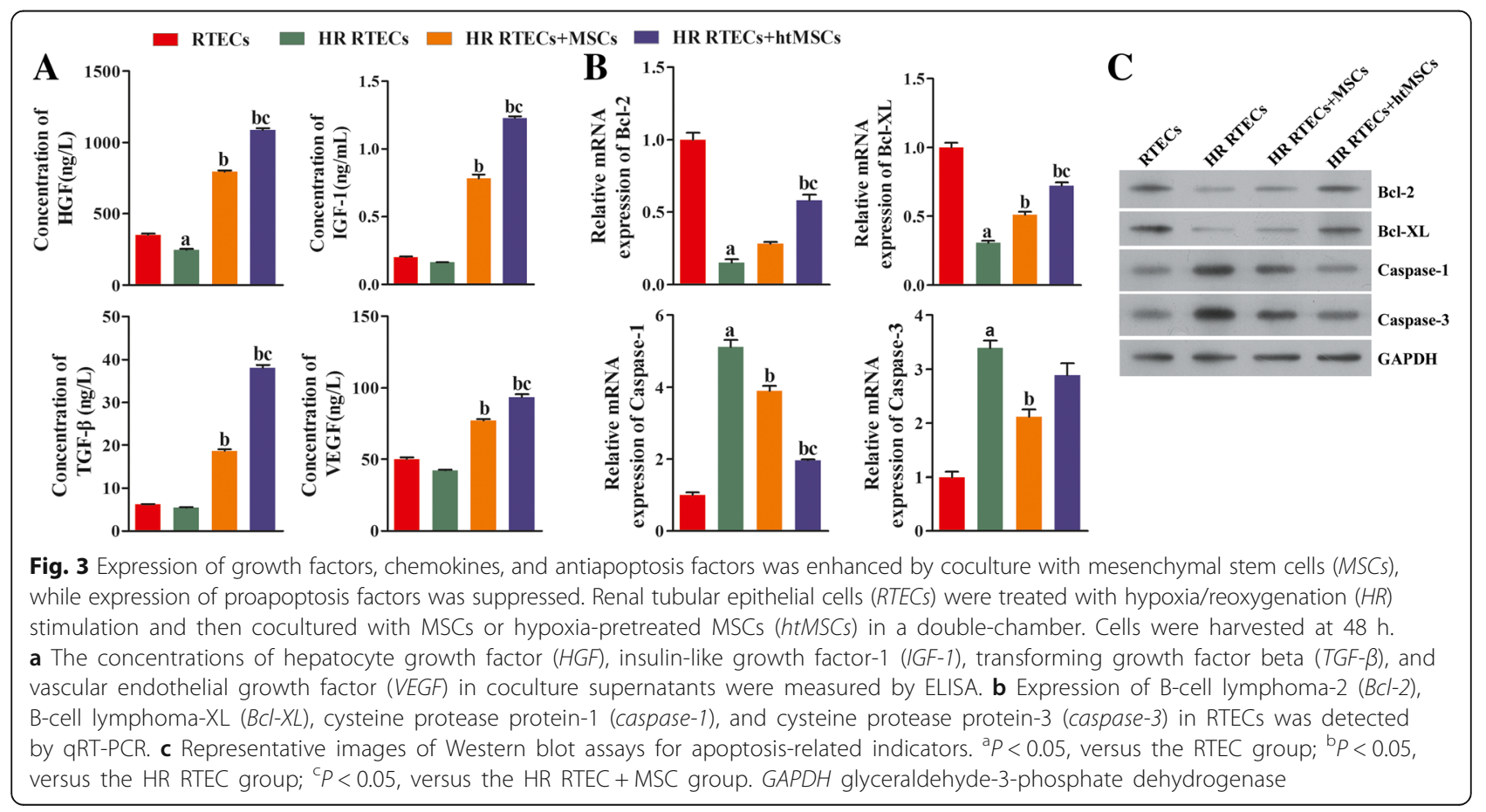


A

5'..GCUAUCUUUCUAYU AACUGAC CAUAA...3, 5'..G - - - UCUUUCUAUU AACUGAC CAUAC.. 5'..GCUAUCUUUCUAUU AACUGAC CAUAA... 5'..GCUAUCUUUCUAUU AaCUGAC CAUAa...' 5'..G - - - UCUUUCUAUU AACUGAC CAUCC...3' 3, aCcCCauahacuguUUGaCUG U 5 , mmu-miR-223-3p

\section{C}

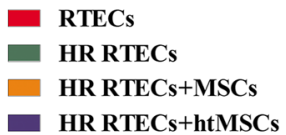

政

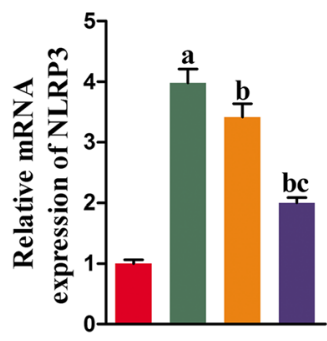

NLRP3

GAPDH

$\mathbf{E}$
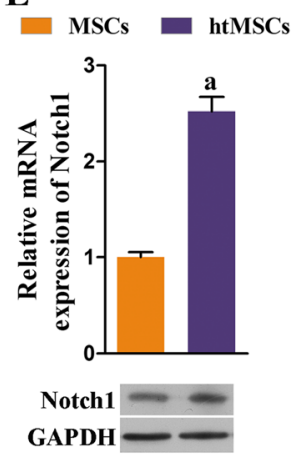
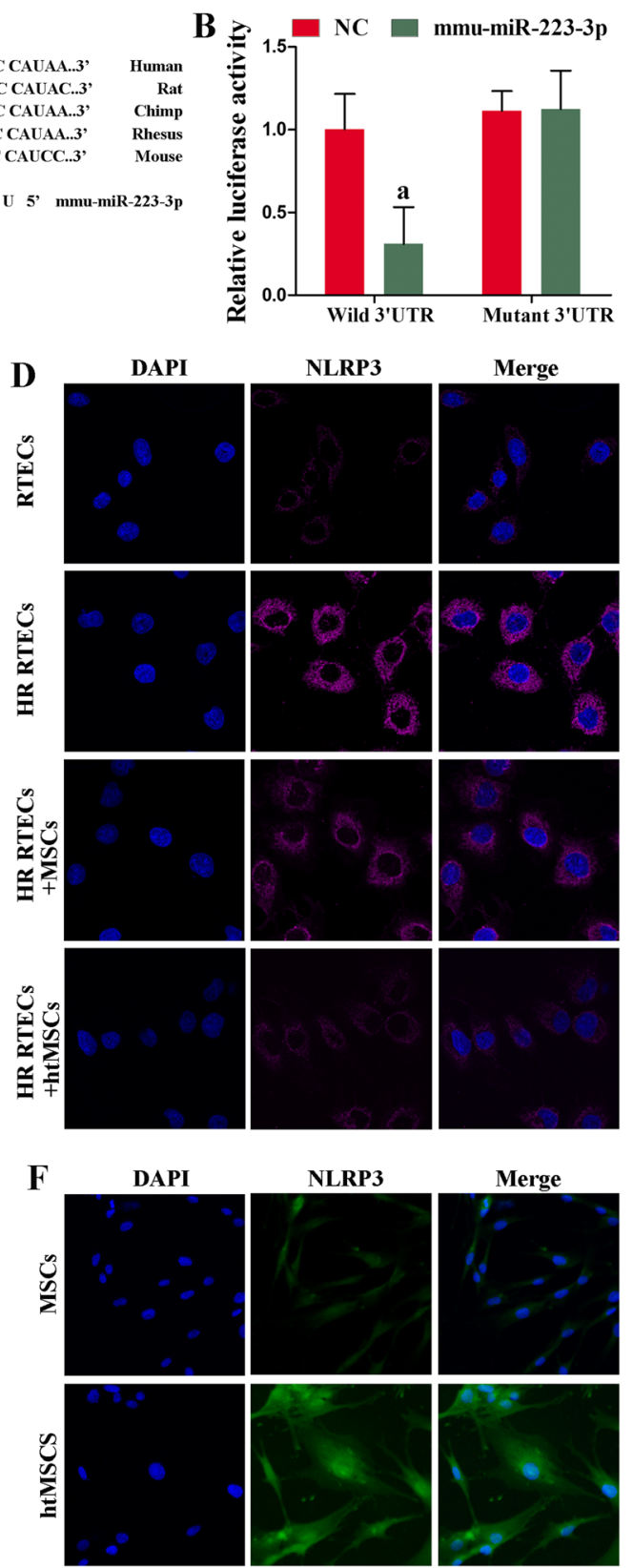

Fig. 4 Expression of NLRP3 was downregulated in renal tubular epithelial cells (RTECS) cocultured with mesenchymal stem cells (MSCS), and Notch signaling in MSCs was initiated by hypoxia pretreatment. a The effect of miR-223 on NLRP3 expression. The binding site was between miR-223 and the $3^{\prime}-U T R$ of NLRP3 mRNA. $\mathbf{b}$ Dual luciferase assay. ${ }^{*} P<0.05$, versus the NC group. c RTECs were treated with hypoxia/reoxygenation $(H R)$ stimulation and then cocultured with MSCs or hypoxia-pretreated MSCs (htMSCs) in a double-chamber. Cells were harvested at 48 h. Expression of NLRP3 in RTECs was detected by qRT-PCR. ${ }^{a} P<0.05$, versus the RTEC group; ${ }^{b} p<0.05$, versus the HR RTEC group; ${ }^{c} P<0.05$, versus the HR RTEC+ MSC group. $\mathbf{d}$ Representative images from the immunofluorescence assays used to detect NLRP3 in RTECs ( $\times 400$ magnification). e Expression of Notch1 in MSCs was detected by qRT-PCR. ${ }^{a} P<0.05$, versus the normal cultured MSCs. f Representative images from immunofluorescence assays used to detect Notch1 in MSCs (×400 magnification). DAPI 4,6-diamino-2-phenyl indole, GAPDH glyceraldehyde-3-phosphate dehydrogenase, NC negative control, NLRP3 NLR family-pyrin domain containing 3, UTR untranslated region

our findings from the in vitro assays, which showed that induction with $\mathrm{I} / \mathrm{R}$ could initiate the innate production of miR-223. In accord with the results from in vitro assays, the expression levels of antiapoptosis molecules $\mathrm{Bcl}-2$ and $\mathrm{Bcl}-\mathrm{XL}$ were enhanced, while those of several proapoptosis molecules (caspase-1, caspase-3, and NLRP3) were suppressed in the groups treated with functional MSCs (Fig. 8b and c). Details of the histological changes seen in the mouse models are shown in Fig. 9. H\&E staining revealed that administration of 
A

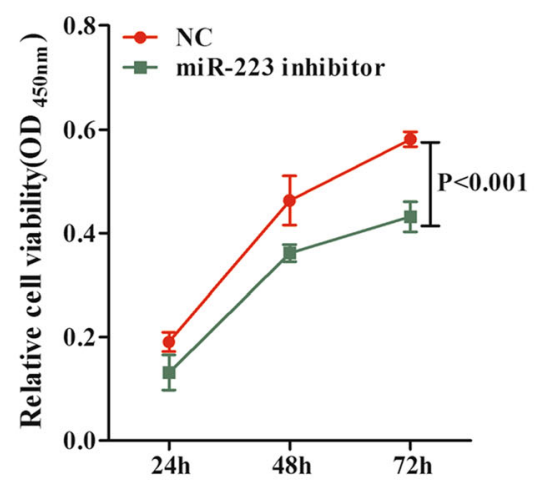

B

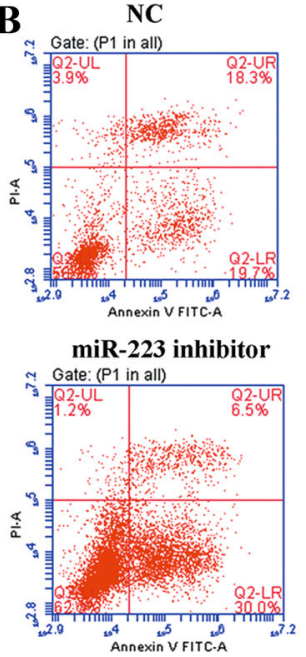

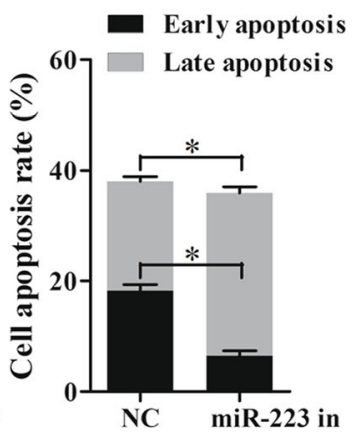

Fig. 5 Downregulation of miR-223 in MSCs decreased cell viability and accelerated the apoptosis process in RTECs. All RTECs were treated with hypoxia/reoxygenation stimulation. The MSCs had been pretreated with a miR-223 inhibitor. The RTECs and MSCs were then cocultured in a double-chamber. a RTEC viability was measured with the CCK-8 assay. $\mathbf{b}$ Representative images of apoptotic RTECs at $48 \mathrm{~h}$ as detected by flow cytometry, ${ }^{*} P<0.05, n=3$. NC negative control, OD optical density, $P$ I propidium iodide

MSCs with active miR-223 restored cellular structures that had been damaged due to $\mathrm{I} / \mathrm{R}$ induction (Fig. 9a). The degrees of kidney fibrosis were also reduced by administration of functional MSCs (Fig. 9b). Additionally, we also used an immunohistochemical assay to detect
Notch1 expression in the RTECs obtained from our mouse model. Interestingly, both the expression and distribution of Notch1 in the mouse kidneys were increased (Notch1-positive cells were stained brown) (Fig. 9c), which might signify initiation of a self-protection

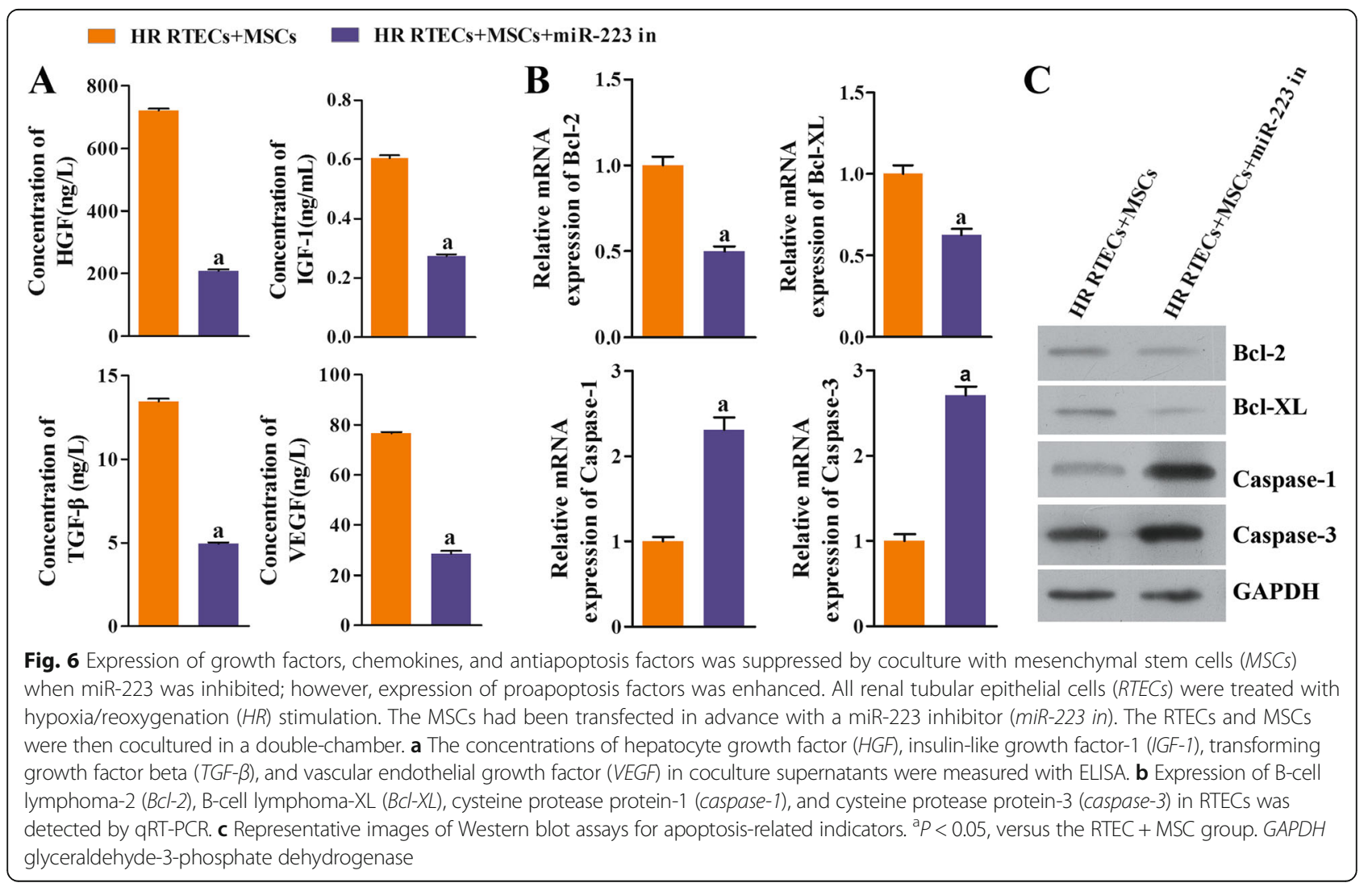



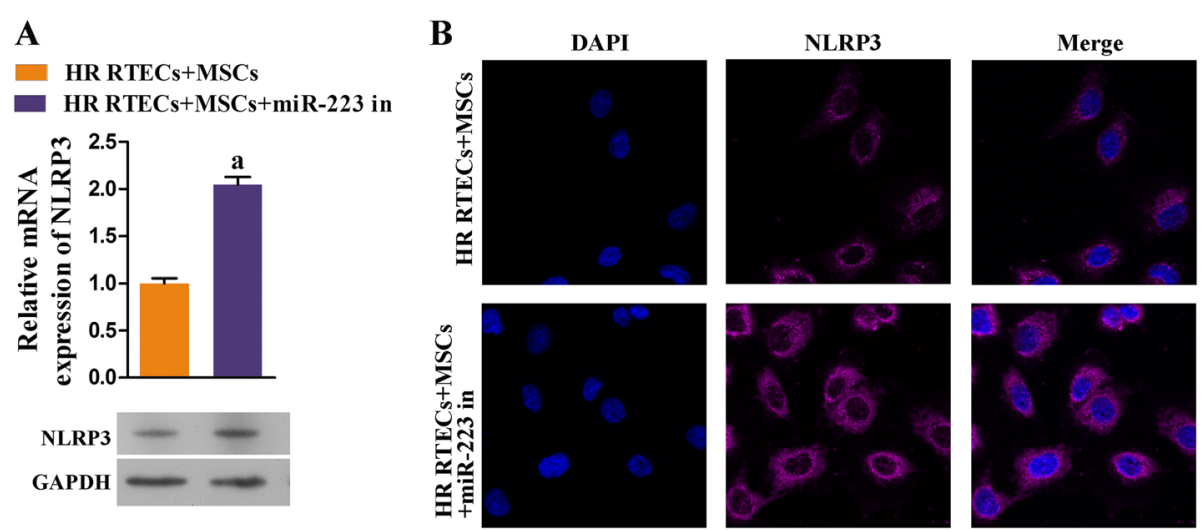

Fig. 7 Expression of NLRP3 in renal tubular epithelial cells (RTECS) was upregulated after coculture with mesenchymal stem cells (MSCS), during which miR-223 was inhibited. All RTECs were treated with hypoxia/reoxygenation (HR) stimulation. The MSCs had been transfected in advance with a miR-223 inhibitor (miR-223 in). The RTECs and MSCs were then cocultured in a double-chamber. a Expression of NLR family-pyrin domain containing-3 (NLRP3) in RTECs was detected by qRT-PCR and Western blot assays. ${ }^{a} P<0.05$, versus the RTEC + MSC group. $\mathbf{b}$ Representative images from the immunofluorescence assay for NLRP3 in RTECS. DAPI 4,6-diamino-2-phenyl indole, GAPDH glyceraldehyde-3-phosphate dehydrogenase

response in I/R-injured kidney tissues. Our TUNEL staining results showed that the numbers of apoptotic RTECs were decreased after treatment with MSCs when compared with those numbers in the $\mathrm{I} / \mathrm{R}$ group. This reduced level of apoptosis in the I/R + MSC group was absent when miR-223 was inhibited. When compared with the levels of apoptosis in the I/R and I/R + MSC groups, a significant inhibition of apoptosis was found after treatment with htMSCs $(P<0.05)$ (Fig. 9d). In a similar manner, results of BUN assays also indicated that treatment with MSCs or htMSCs significantly decreased the levels of BUN, whereas suppression of miR-223 impeded the reduction in BUN induced by MSCs or htMSCs $(P<0.05)$ (Fig. 9e). We also examined the creatinine clearance rates in mice which received the different treatments, and found that the creatinine clearance rates were significantly increased in the $I / R+M S C$ group and were even upregulated in the $I / R+h t M S C$ group (Fig. 9f). Interestingly, downregulation of miR-223 significantly reduced creatinine clearance rates in the $I / R+M S C$ group, indicating that miR-223 plays a role in the ability of MSCs to treat I/R-induced kidney injuries.

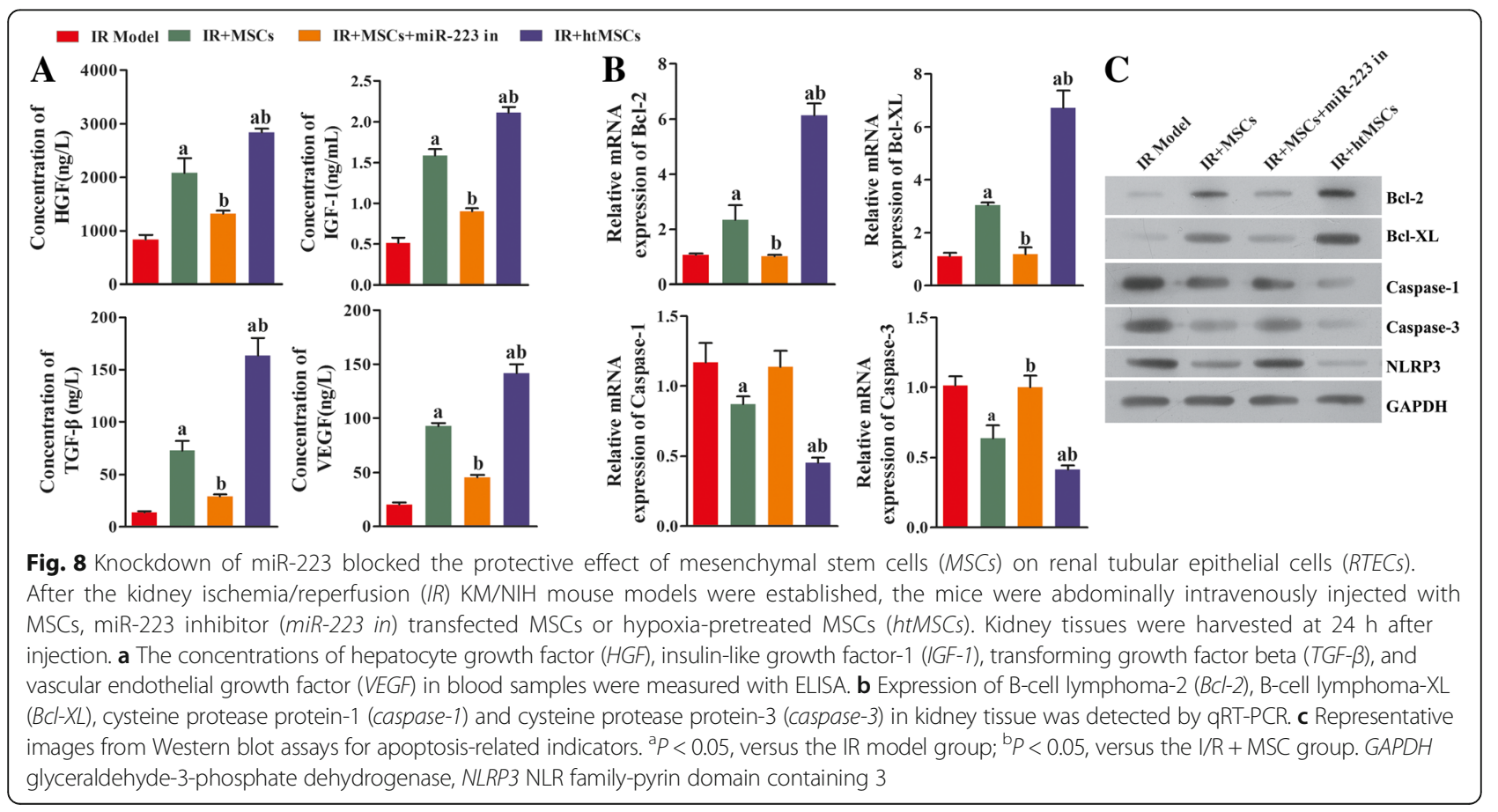




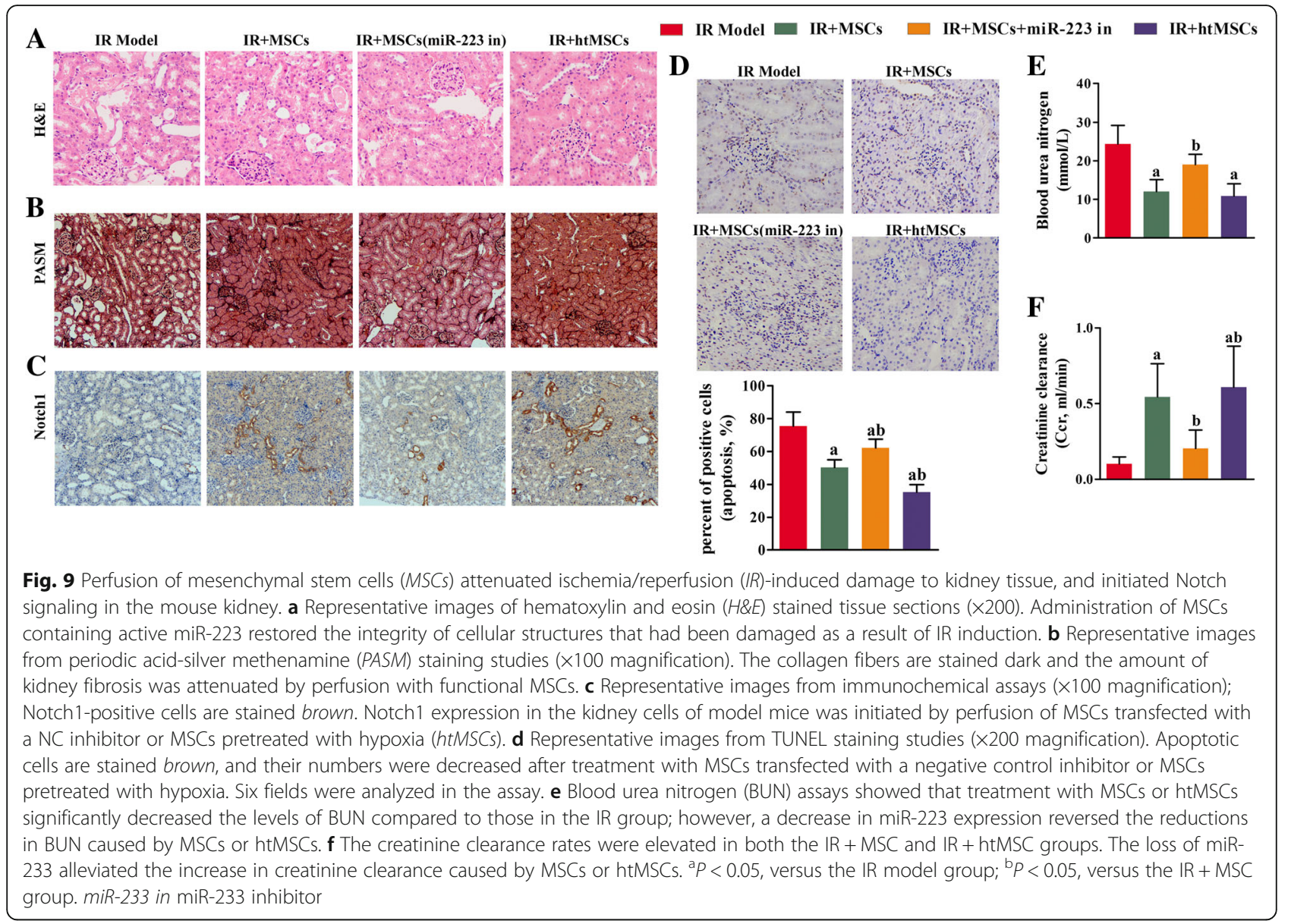

\section{Discussion}

While therapies based on MSC transplantation have been successfully used to alleviate injuries in liver, heart, and renal models $[13,14,24,25]$, the underlying mechanisms involved in the protective effect of MSCs on injured tissues have never been fully understood. In the current study, we demonstrated that administration of MSCs could ameliorate kidney damage induced by I/R, and the related mechanism involved inhibition of NLRP3 activity via activation of miR-223.

MicroRNAs comprise a group of endogenous short noncoding RNAs which modulate the translation of targeted messenger RNAs by binding to the 3'-UTR of the targeted messenger RNA transcripts, thereby leading to RNA degradation and inhibition of protein synthesis [26]. In recent years, numerous associations between renal I/R and specific miRNAs have been identified. Wei et al. [27] found that deletion of Dicer in the proximal tubular epithelium protected kidney tissue from I/R-induced injuries, and this effect was correlated with changes in the expression of specific miRNAs [27]. Moreover, in a unilateral warm renal ischemia model created in mice, nine miRNAs were found to be differentially expressed in the ischemic kidneys when compared with the control kidneys [28]. Our previous studies showed that the protective effect of MSCs on a DCD rat kidney was associated with activation of miR-223, as well as the specific suppression of its targeted gene, NLRP3. Therefore, it was reasonable to hypothesize that miR223 plays a key role in I/R-induced injuries to the kidney, as well as the ability of MSCs to protect kidney tissue. For the following reasons, this hypothesis was validated in the current study: 1) RTECs from an induced $H / R$ model displayed significantly decreased levels of miR-223 expression in vitro; and 2) knockdown of miR223 in MSCs impaired the treatment effect of MSCs on RTECs in vitro, and on renal I/R mice in vivo. A previous study reported that MSCs secrete exosomes capable of transporting miR-223 into nearby cells for the purpose of regulating targeted pathways [24]. In the present study, results obtained after the coculture of MSCs with RTECs without physical contact partially confirmed that idea. After administration of MSCs alone, the levels of miR-223 in RTECs dramatically increased. Moreover, miR-223 appeared to affect the amounts of growth factors and chemokines secreted by MSCs. It was noteworthy that suppression of miR-223 decreased HGF, IGF-1, TGF- $\beta$, and VEGF production both in vitro and 
in vivo. Because secretion of these polypeptides contributes to the ability of MSCs to help increase epithelial proliferation and modulate inflammation or angiogenesis, those polypeptides can be considered as therapeutic candidates for treating renal injuries [29-31]. Taken together, the central role played by miR-223 during the onset of a renal $I / R$ injury was affirmed. Thus, the upregulation of miR-223 in kidney tissues by therapeutic modalities such as MSCs is a promising method for treating renal failure induced by an $\mathrm{I} / \mathrm{R}$ process.

Our current study investigated the mechanism by which miR-223 functions as a key mediator between MSCs and RTECs. Previous studies have shown that Notch1 receptors are expressed in MSCs, which were proven to serve as upstream regulators of miR-223 $[21,22]$. Thus we investigated the relationship between Notch1 expression and miR-223 levels in MSCs. As it has been demonstrated that hypoxiatreated MSCs can initiate Notch signaling [32], we found that the levels of miR-223 expression in MSCs were significantly increased. Those results proved the positive correlation between miR-223 expression and activation of Notch1.

Thereafter, we investigated the levels of miR-223 target gene (NLRP3) expression in both RTECs and kidney tissue $[20,33]$. Nod-like receptors are crucial signaling regulators involved in the formation of intracellular macromolecular complexes, and contribute to host immune responses [34]. Shigeoka et al. [19] showed that NLRP3 promoted tissue injury by exerting a direct effect on the renal tubular epithelium in an inflammasomeindependent manner [19]. Our study showed that upregulated levels of NLRP3 were closely associated with induction of an $I / R$ injury both in vitro and in vivo. At the molecular level, enhanced NLRP3 activity contributed to the onset of inflammation by increasing caspase- 1 levels [35], and to the initiation of mitochondrial pathwaymediated apoptosis by increasing caspase- 3 levels and decreasing Bcl-2 and Bcl-XL levels [36]. Administration of MSCs suppressed the function of NLRP3 both in RTECs and kidney tissues, whereas knockdown of miR223 blocked the effect of MSCs. These findings indicate that MSCs protect RTECs and kidney tissue by inhibiting NLRP3 via miR-223, and this effect was even more evident when Notch signaling in MSCs was activated by hypoxia treatment. Additionally, during the immunochemical detection of Notch1 in mouse kidney tissues, it was found that Notch1 levels in the MSCs of I/R-treated mice increased in synchrony with upregulation of miR223 in the corresponding samples. This finding suggests activation of a self-protective signaling pathway in I/Rinjured kidneys. Data from apoptosis, BUN, and creatinine clearance studies also suggested a significant role for miR-223 in the alleviation of kidney $I / R$ injuries by implanted MSCs. However, a determination of whether Notch1 activation in kidney tissue represents a feedback response to miR-223 or occurs due to direct contact with MSCs in vivo requires further investigation.

\section{Conclusions}

Our findings demonstrate that MSCs ameliorated I/R-induced renal failure via a paracrine mechanism. The exosomal miR-223 secreted by MSCs was transferred into RTECs and attenuated damage to renal cells after NLRP signaling was suppressed. Moreover, the protective effect of MSCs on kidney tissue was enhanced by hypoxia pretreatment, which increased miR-223 levels by activating Notch1 expression. Our studies with an in vivo mouse model show that the therapeutic effect of MSCs on I/Rinduced renal injuries was reduced by suppression of the miR-223/Notch signaling pathway. When taken together, our data show that MSCs can attenuate renal I/R-induced injuries by transporting miR-223 into kidney cells and inhibiting NLRP3.

\section{Abbreviations \\ BCl-2: B cell leukemia/lymphoma 2; BCl-XL: B cell leukemia/lymphoma 2 -like 1; BUN: Blood urea nitrogen; Caspase-1/3: Cysteinyl aspartate specific proteinase-1/3; DAPI: 4,6-Diamino-2-phenyl indole; DMEM: Dulbecco's modified Eagle's medium; ELISA: Enzyme-linked immunosorbent assay; FBS: Fetal bovine serum; GAPDH: Glyceraldehyde-3-phosphate dehydrogenase; H\&E: Hematoxylin and eosin; H/R: Hypoxia/reoxygenation; HGF: Hepatocyte growth factor; htMSC: Hypoxia-pretreated mesenchymal stem cell; I/R: Ischemia/reperfusion; IGF-1: Insulin-like growth factor-1; MSC: Mesenchymal stem cell; NLRP3: NLR family-pyrin domain containing 3; PASM: Periodic acid-silver methenamine; PBS: Phosphate-buffered saline; qRT-PCR: Quantitative real-time polymerase chain reaction; RTEC: Renal tubular epithelial cell; TGF- $\beta$ : Transforming growth factor beta; \\ TUNEL: Terminal deoxynucleotidyl transferase-mediated nick-end labeling; UTR: Untranslated region; VEGF: Vascular endothelial growth factor}

\section{Acknowledgements}

We are grateful to our collaborators in the multiple disciplinary teams at the Eastern Campus of First Affiliated Hospital, Sun Yat-sen University.

\section{Funding}

This study was supported by grants from the Science and Technology Planning Project of Guangdong province (2014A020212719).

\section{Availability of data and materials}

Yes.

\section{Authors' contributions}

Conception and design: XY and XW. Analysis and interpretation: XY, XW, CC, $\mathrm{JZ}$, and $\mathrm{MH}$. Drafting the manuscript and checking for important intellectual content: XY. All authors critically read and revised the manuscript, and approved its submission for publication.

\section{Competing interests}

The authors declare that they have no competing interests.

\section{Consent for publication}

All authors have reviewed the manuscript and approved its submission for publication.

\section{Ethics approval and consent to participate}

The protocol for this study was approved by the Animal Care \& Welfare Committee of The First Affiliated Hospital of Sun Yat-sen University (No. B2016-0221). 


\section{Publisher's Note}

Springer Nature remains neutral with regard to jurisdictional claims in published maps and institutional affiliations.

Received: 17 March 2017 Revised: 24 May 2017

Accepted: 25 May 2017 Published online: 15 June 2017

\section{References}

1. Takada M, Nadeau KC, Shaw GD, Tilney NL. Prevention of late renal changes after initial ischemia/reperfusion injury by blocking early selectin binding. Transplantation. 1997;64(11):1520-5

2. Matin SF, Novick AC. Renal dysfunction associated with staged bilateral partial nephrectomy: the importance of operative positioning. J Urol. 2001; 165(3):880-1.

3. Kazmers A, Jacobs L, Perkins A. The impact of complications after vascular surgery in Veterans Affairs Medical Centers. J Surg Res. 1997;67(1):62-6.

4. Krysztopik RJ, Bentley FR, Spain DA, Wilson MA, et al. Free radical scavenging by lazaroids improves renal blood flow during sepsis. Surgery. 1996;120(4):657-62.

5. Koo DD, Welsh Kl, West NE, Channon KM, et al. Endothelial cell protection against ischemia/reperfusion injury by lecithinized superoxide dismutase. Kidney Int. 2001;60(2):786-96

6. DuBose Jr TD, Warnock DG, Mehta RL, Bonventre JV et al. Acute renal failure in the 21st century: recommendations for management and outcomes assessment. Am J Kidney Dis. 1997;29(5):793-9.

7. Guo JK, Cantley LG. Cellular maintenance and repair of the kidney. Annu Rev Physiol. 2010;72:357-76.

8. Humphreys BD, Bonventre JV. Mesenchymal stem cells in acute kidney injury. Annu Rev Med. 2008;59:311-25.

9. Togel F, Weiss $K$, Yang $Y, H u Z$, et al. Vasculotropic, paracrine actions of infused mesenchymal stem cells are important to the recovery from acute kidney injury. Am J Physiol. 2007:292(5):F1626-35.

10. Chen YT, Sun CK, Lin YC, Chang LT, et al. Adipose-derived mesenchymal stem cell protects kidneys against ischemia-reperfusion injury through suppressing oxidative stress and inflammatory reaction. J Transl Med. 2011 9.51.

11. Caplan Al. Mesenchymal stem cells. J Orthop Res. 1991;9(5):641-50.

12. Semedo P, Wang PM, Andreucci TH, Cenedeze MA, et al. Mesenchymal stem cells ameliorate tissue damages triggered by renal ischemia and reperfusion injury. Transplant Proc. 2007;39(2):421-3.

13. Rosova I, Dao M, Capoccia B, Link D, et al. Hypoxic preconditioning results in increased motility and improved therapeutic potential of human mesenchymal stem cells. Stem Cells. 2008:26(8):2173-82.

14. Lange $\mathrm{C}$, Togel $\mathrm{F}$, Ittrich $\mathrm{H}$, Clayton $\mathrm{F}$, et al. Administered mesenchymal stem cells enhance recovery from ischemia/reperfusion-induced acute renal failure in rats. Kidney Int. 2005;68(4):1613-7.

15. Bi B, Schmitt R, Israilova M, Nishio H, et al. Stromal cells protect against acute tubular injury via an endocrine effect. J Am Soc Nephrol. 2007;18(9): 2486-96.

16. Meirelles Lda S, Fontes AM, Covas DT, Caplan Al. Mechanisms involved in the therapeutic properties of mesenchymal stem cells. Cytokine Growth Factor Rev. 2009;20(5-6):419-27.

17. Tögel FE, Westenfelder C. Mesenchymal stem cells: a new therapeutic tool for AKI. Nat Rev Nephrol. 2010;6(3):179-83.

18. Vilaysane A, Chun J, Seamone ME, Wang W, et al. The NLRP3 inflammasome promotes renal inflammation and contributes to CKD. J Am Soc Nephrol. 2010;21(10):1732-44

19. Shigeoka AA, Mueller JL, Kambo A, Mathison JC, et al. An inflammasomeindependent role for epithelial-expressed Nlrp3 in renal ischemiareperfusion injury. J Immunol. 2010;185(10):6277-85.

20. Bauernfeind F, Rieger A, Schildberg FA, Knolle PA, et al. NLRP3 inflammasome activity is negatively controlled by miR-223. J Immunol. 2012;189(8):4175-81.

21. Li H, Yu B, Zhang Y, Pan Z, et al. Jagged1 protein enhances the differentiation of mesenchymal stem cells into cardiomyocytes. Biochem Biophys Res Commun. 2006:341(2):320-5.

22. Kumar V, Palermo R, Talora C, Campese AF, et al. Notch and NF-kB signaling pathways regulate miR-223/FBXW7 axis in T-cell acute lymphoblastic leukemia. Leukemia. 2014;28(12):2324-35.
23. Ogawa S, Gerlach H, Esposito C, Pasagian-Macaulay A, et al. Hypoxia modulates the barrier and coagulant function of cultured bovine endothelium: increased monolayer permeability and induction of procoagulant properties. J Clin Investig. 1990;85(4):1090-8.

24. Wang X, Gu H, Qin D, Yang L, et al. Exosomal miR-223 contributes to mesenchymal stem cell-elicited cardioprotection in polymicrobial sepsis. Sci Rep. 2015;5:13721.

25. Ji JF, He BP, Dheen ST, Tay SS. Interactions of chemokines and chemokine receptors mediate the migration of mesenchymal stem cells to the impaired site in the brain after hypoglossal nerve injury. Stem Cells. 2004; 22(3):415-27.

26. Fiedler J, Jazbutyte $V$, Kirchmaier BC, Gupta SK, et al. MicroRNA-24 regulates vascularity after myocardial infarction. Circulation. 2011:124(6):720-30.

27. Wei Q, Bhatt K, He HZ, Mi QS, et al. Targeted deletion of Dicer from proximal tubules protects against renal ischemia-reperfusion injury. J Am Soc Nephrol. 2010;21(5):756-61.

28. Godwin JG, Ge X, Stephan K, Jurisch A, et al. Identification of a microRNA signature of renal ischemia reperfusion injury. Proc Natl Acad Sci U S A. 2010;107(32):14339-44.

29. Hirschberg R, Adler S. Insulin-like growth factor system and the kidney: physiology, pathophysiology, and therapeutic implications. Am J Kidney Dis. 1998:31(6):901-19.

30. Liu Y. Hepatocyte growth factor and the kidney. Curr Opin Nephro Hypertens. 2002:11(1):23-30.

31. Chen H, Li D, Saldeen T, Mehta JL. TGF-beta 1 attenuates myocardial ischemia-reperfusion injury via inhibition of upregulation of MMP-1. Am J Physiol. 2003;284(5):H1612-7.

32. Eliasz S, Liang S, Chen Y, De Marco MA, et al. Notch-1 stimulates survival of lung adenocarcinoma cells during hypoxia by activating the IGF-1R pathway. Oncogene. 2010;29(17):2488-98.

33. Shao BZ, Xu ZO, Han BZ, Su DF, et al. NLRP3 inflammasome and its inhibitors: a review. Front Pharmacol. 2015;6:262.

34. Geddes K, Magalhaes JG, Girardin SE. Unleashing the therapeutic potential of NOD-like receptors. Nat Rev Drug Discov. 2009;8(6):465-79.

35. Agostini L, Martinon F, Burns K, McDermott MF, et al. NALP3 forms an IL-1 beta-processing inflammasome with increased activity in Muckle-Wells autoinflammatory disorder. Immunity. 2004;20(3):319-25.

36. Jin Z, El-Deiry WS. Overview of cell death signaling pathways. Cancer Biol Ther. 2005:4(2):139-63.

\section{Submit your next manuscript to BioMed Central and we will help you at every step:}

- We accept pre-submission inquiries

- Our selector tool helps you to find the most relevant journal

- We provide round the clock customer support

- Convenient online submission

- Thorough peer review

- Inclusion in PubMed and all major indexing services

- Maximum visibility for your research

Submit your manuscript at www.biomedcentral.com/submit 